\title{
Reflectivity Parameter Extraction from RADAR Images Using Back Propagation Algorithms
}

\author{
P. Anil Kumar, B. Anuradha \\ Department of ECE, S V University College of Engineering, India
}

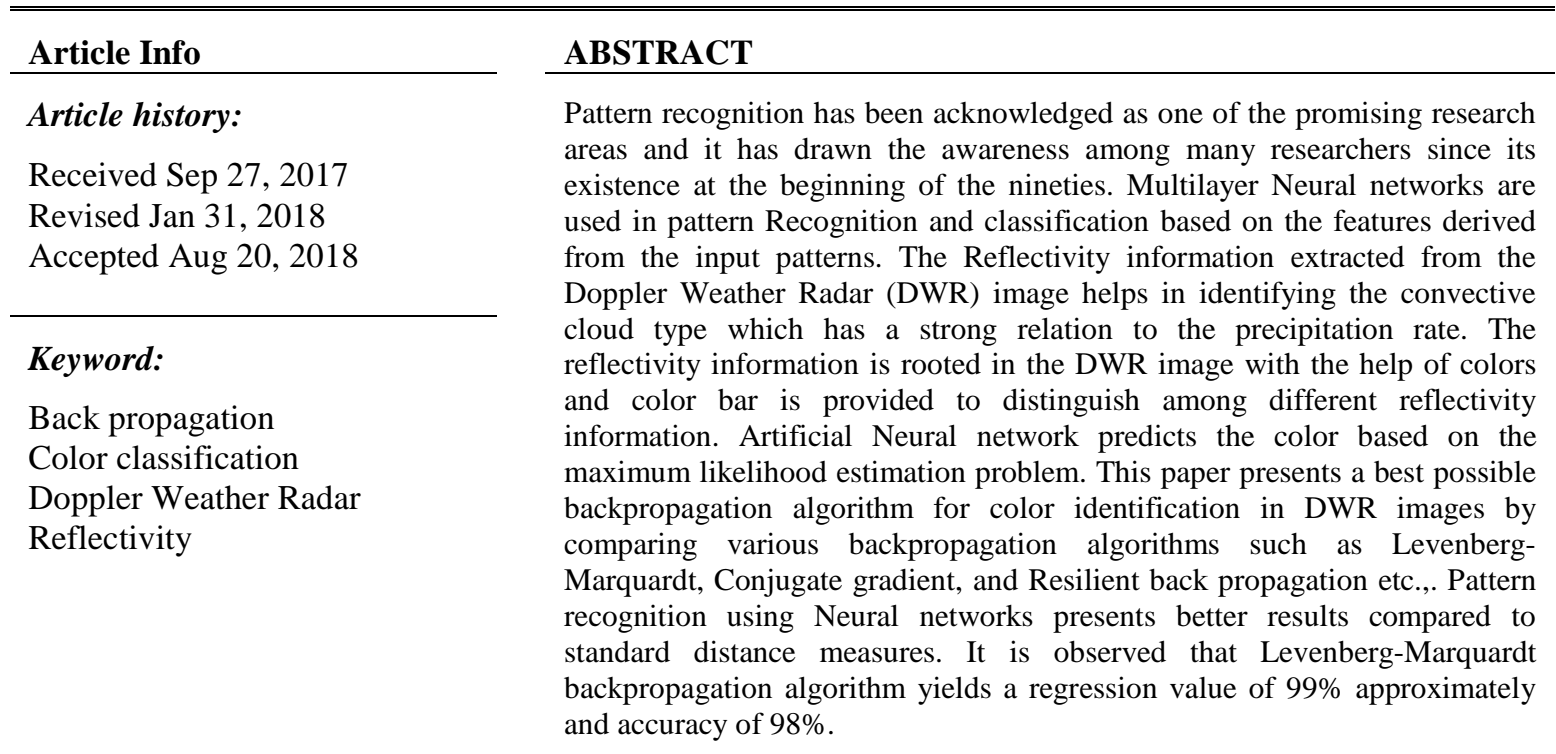

Copyright (C) 2018 Institute of Advanced Engineering and Science. All rights reserved.

Corresponding Author:

P. Anil Kumar,

Department of ECE,

S V University College of Engineering, Tirupati, A.P., India.

E-mail: anilkumar417@gmail.com

\section{INTRODUCTION}

Clouds are helpful in maintaining the earth's energy balance. Clouds are classified based on their structure and height in the atmosphere which influences the radiation budget in different ways. Apart from cloud height in the atmosphere, Cloud optical thickness plays a crucial role in the cloud classification. Cloud optical thickness is directly related to the reflectivity of the DWR image [1]. The higher the reflectivity value, the thicker the cloud. The detection of convective clouds based on the reflectivity parameter from the MAX$\mathrm{Z}$ product of DWR images plays a key role in estimating the amount of precipitation intensity. The convective clouds are classified based on the reflectivity parameter by professional humans at the Indian Meteorological Department (IMD), which will sometimes lead to contradicting the results due to man-made errors. Hence, retrieval of reflectivity information from the DWR images without human intervention is essential in this field. Many researchers in this field work on the DWR raw and processed data rather than the image to exploit the relation between reflectivity and rainfall rate. The processed products available with the IMD are Reflectivity, Surface Rainfall Intensity (SRI), Precipitation Accumulation (PAC), Plan Position Indicator (PPI), Plan Position Indicator - Close Range and Volume Velocity Processing.

Color images communicate a large amount of information rather than the grayscale image or the binary image [2, 3]. Color images are preferred over gray images because they convey more information with minimal effort [4]. The DWR images convey information about Reflectivity, Surface Rainfall Intensity, Precipitation Accumulation, Wind direction, and Intensity. The value of the product at a particular location 
and time is identified with the help of color bar provided on the right hand side of the image. A large number of distance metrics are available in literature till now for color matching.

Pattern recognition is a branch of machine learning, which focuses on recognizing different patterns. Pattern Recognition provides the solution to speech recognition, recognition of handwritten characters, face recognition and medical diagnosis. Artificial Neural Networks (ANN) are useful where the limits between different patterns are not defined precisely [5].

In supervised learning, input patterns and its corresponding output patterns are provided for learning which is used to adjust the neuron weights whereas, unsupervised learning no training samples are provided. In pattern recognition, outputs are classified based on input sets, bias weights and neuron weights. Supervised classification finds its application in the area of curve fitting, time series prediction, etc. Unsupervised classification finds its application in the area of clustering. The reinforcement learning is a type of machine learning which aims at maximizing the performance for a specific problem of context. The output of the network depends on the past experiences which is a trial and error approach.

This paper focuses on color identification of DWR images for reflectivity extraction using Artificial Neural Networks. In Section 2, the basics of Artificial Neural Networks are discussed. In Section 3 different Back Propagation Neural Network methods are discussed. In section 4, Research Method to extract reflectivity from DWR images is discussed. In section 5 results and discussion are discussed, followed by conclusions and by the references.

\section{ARTIFICIAL NEURAL NETWORKS}

ANN functions in a similar way to that of the brain. The basic structure of Artificial Neural Network (ANN) is shown in Figure 1. The network shown in Figure 1 has $\mathrm{m}$ input samples, $\mathrm{n}$ output samples, and $\mathrm{k}$ hidden layers. The network is provided with sample inputs and it's corresponding sample outputs to train and adjust the output and hidden layer neuron weights [6]. Each input signal $\mathrm{x} 1, \mathrm{x} 2, \ldots \mathrm{xn}$ is modeled by some weight values w1, w2, ... wn. The sum of the product of the inputs and the weights is applied to a thresholding function (also called as activation function), which model the output. The constant difference between actual value and the desired value is adjusted using a bias value. The basic ANN equations are shown in Equation 1 and 2. There are a wide variety of activation function such as binary threshold function (also referred to as Heaviside function), Fermi function (logistic function), hyperbolic tangent etc., available in the literature. The thresholding function will be chosen depending on the nature of the problem.

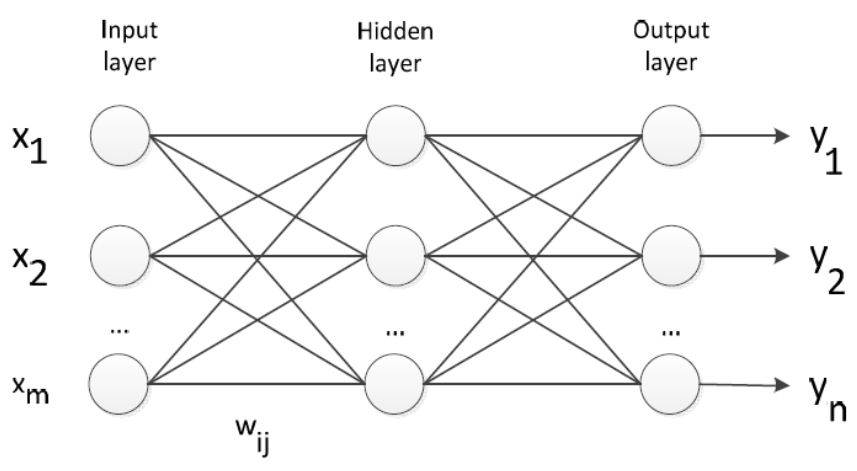

Figure 1. Artificial Neural Networks

Net $=\sum_{i=1}^{n} x_{i} * w_{i}+$ bias

Output=f (Net)

ANN has proven to be an efficient alternative to traditional methods of distance measures [7, 8, 9]. The backpropagation learning algorithm is used for training multilayer perceptrons (MLP). The MLP consists of a set of an input layer for receiving inputs, one or more hidden layers for computation, and an output layer for presenting the output. The signal is applied at the input layer which propagates to the output through the hidden layer [10, 11]. Input layer communicates with the external world and presents a pattern to the network. This process is called as excitation. The output layer presents a pattern to the external world. The 
number of outputs depending on the type of problem. Hidden layer acts as an intermediate layer between input and output. Hidden layers are not required for linear separable problems. Depends on the complexity of the problem, the number of hidden layers is decided.

The neural network has to be provided with a sufficient number of input samples for training. Underfitting is a condition when the number of input samples is less than the minimum number of input samples required for training. When the number of input samples is more than the minimum number of input samples required for training leads to overfitting. The number of hidden layer neurons required for a problem is $2 / 3$ rd the sum of the number of input and output neurons [12]. In order to save computation time and memory, the number of hidden layers in first and second layer should be almost same. There are different algorithms used in this paper for training the neural network is Levenberg-Marquardt, Conjugate gradient, and resilient backpropagation algorithm [13]. The error of the network is defined as the difference between desired target and network response. The performance of the network is evaluated using different metrics such as mean square error (RMSE), Mean Absolute Error (MAE), Mean Absolute Deviation (MAD) as shown in Equation 3, 4 and 5. The main focus of this back propagation algorithm is to reduce the global error as minimum as possible.

$$
\begin{aligned}
& \mathrm{RMSE}=\sqrt{\frac{\sum_{i=1}^{N}\left(y_{i}-d_{i}\right)^{2}}{N}} \\
& \mathrm{MAE}=\frac{1}{N} \sum_{i=1}^{N}\left|y_{i}-d_{i}\right| \\
& \mathrm{MAD}=\frac{1}{N} \sum_{i=1}^{N}\left|y_{i}-\overline{y_{i}}\right|
\end{aligned}
$$

\section{THE DIFFERENT BACK PROPAGATION NEURAL NETWORK METHODS}

There are various Backpropagation algorithms supported in the literature. Of them, Gradient Descent (GD), Gradient Descent with Momentum (GDM), Variable Learning Rate with Momentum (GDX), Conjugate Gradient (CGP), Quasi-Newton (BFGS), Levenberg-Marquardt (LM), and Resilient back propagation $(\mathrm{RB})$ are used to adjust the weights of the Neural network.

In the gradient descent Back Propagation algorithm, bias weights and network weights are updated in the direction of negative gradient performance function $[14,15]$. The parameter $\eta$ is the learning rate parameter which has a direct influence on the training the network. Gk is the error gradient with respect to the weight vector. The updated weight vector is given in Equation 6 [16]. Gradient Descent suffers from the shallow local minimum. GDM can skip such minimum values by updating the weight values equal to the sum of modified weight in Gradient descent and the fraction of previous weight values as given in Equation 7. The parameter $\mu$ is the coefficient of momentum and it varies between 0 and 1 .

$$
\begin{aligned}
& \mathrm{Wk}+1=\mathrm{Wk}-\eta * \mathrm{Gk} \\
& \mathrm{Wk}+1=\mathrm{Wk}-\eta * \mathrm{Gk}-\mu * \mathrm{Wk}-1
\end{aligned}
$$

The GD and GDM method suffers from the problem of low convergence rate. The learning rate parameter value has a direct relation to the convergence. As the learning rate increases convergence value increases. The algorithm takes a long time to converge if the value of the learning rate parameter is high and it leads to an unstable network. To overcome this problem, variable learning rate backpropagation with momentum is used. In this algorithm, the value of $\eta$ is large initially and it decreases as time progresses. The weight adjustment in GDX is given by equation 8.

$$
\mathrm{Wk}+1=\mathrm{Wk}-\eta \mathrm{k}+1 * \mathrm{gk}+\mu * \mathrm{Wk}-1
$$

The methods discussed till now uses the steepest descent method which works at the direction of the negative gradient for modifying the neuron weights. The convergence rate of these methods is very slow. In order to improve the convergence rate, the conjugate direction of the search is preferred over steepest descent method. Conjugate gradient descent back-propagation algorithm (CGD-BP) is used for training purpose. In CGP search is performed along the conjugate gradient direction which will minimize the cost function along the line by adjusting the step size. The weight update in the conjugate gradient method is given in equation $9[17]$. The direction of Conjugate gradient search is given in equation $10 . \beta \mathrm{k}$ is the ratio of norm squared of the current gradient to norm squared of the previous gradient as shown in the equation in 11. 


$$
\begin{aligned}
& \text { Wk }+1=\mathrm{Wk}+\eta * \mathrm{Pk} \\
& \text { Where, } \mathrm{Pk}=-\mathrm{gk}+\beta \mathrm{k} * \mathrm{Pk}-1 \\
& \beta_{\mathrm{k}}=\frac{\Delta \mathrm{g}_{\mathrm{k}-1}^{\mathrm{T}} * \mathrm{~g}_{\mathrm{k}}}{\mathrm{g}_{\mathrm{k}-1}^{\mathrm{T}} * \mathrm{~g}_{\mathrm{k}-1}}
\end{aligned}
$$

Levenberg-Marquardt algorithm aimed at speeding up the training without computing the Hessian matrix directly. The Hessian matrix is computed using the Jacobian matrix as shown in Equation 12 . The principal diagonal elements of Hessian matrix are larger than zero. The weight update rule in the LevenbergMarquardt algorithm is presented in Equation 13.

$$
\begin{aligned}
& \mathrm{H}=\mathrm{JT} \mathrm{J}+\mu \mathrm{I} \\
& \mathrm{Wk}+1=\mathrm{Wk}-(\mathrm{JkT} \mathrm{Jk}+\mu \mathrm{I})-1 \mathrm{Jk} \text { ek }
\end{aligned}
$$

The Levenberg-Marquardt switches between steepest descent algorithm and Gauss Newton algorithm during the training phase. The convergence rate of the Gauss Newton method is fast and unstable, whereas, Levenberg-Marquardt overcomes the problem instability by maintaining the convergence rate fast. A Gauss Newton algorithm is used when the coefficient $\mu$ is very small. The Steepest Descent method is used when $\mu$ is very large. The relation between learning rate $\eta$ and the combination coefficient $\mu$ is given by the following relation as shown in Equation 14.

$$
\eta=1 / \mu
$$

The activation function typically used in a multilayer network is a sigmoid transfer function. The primary role of activation function is to compress the infinite input range to a finite output range. For higher values of inputs, the slope of the activation function approximates zero. This creates a problem while training multilayer networks since the gradients have a small magnitude. To eliminate these effects, resilient backpropagation training algorithm is used. Sign of the derivative plays a crucial role in the weight update rather than the magnitude of the derivative. If the derivative of the performance function for two successive iterations has the same sign, then the weight update value and bias values will be increased otherwise it has the decreasing pattern. If the derivative is zero, then there is no need to update the weights.

\section{RESEARCH METHOD}

The present work focuses on extracting the reflectivity values from the DWR image. The similar approach is used to extract the SRI and PAC from the DWR images. The images obtained from DWR MAX (Z) product of IMD Chennai contains the Reflectivity information about convective clouds with Chennai as a center and spans around 250Km circular area. A sample DWR MAX (Z) image captured on 19th July 2017 is shown in Figure 2 [18]. The reflectivity information on a particular location on the DWR image is indicated using 17 different colors. The color and its corresponding reflectivity are provided on the right side of the image [19, 20]. The reflectivity value greater than $60 \mathrm{dbZ}$ indicates strong precipitation and hail. The DWR image contains the reflectivity values from $20 \mathrm{dBZ}$ to $65 \mathrm{dBZ}$.

The horizontal, vertical resolution of the DWR image is $1 \mathrm{Km} / \mathrm{Pixel}, 0.089 \mathrm{Km} /$ pixel respectively. The DWR image covers a circular geographical area of $250 \mathrm{Km}$ radius with Chennai as the center. The color information provided on the top of the image provides the maximum value of reflectivity seen along the line from north to south. The color information provided on the right of the image provides the maximum value of reflectivity seen along the line from east to west. The range of convective cloud spans from $0.1 \mathrm{Km}$ to 18 Kms. The DWR image is processed to extract the convective information by eliminating the static background image. The steps involved in preprocessing the DWR image are noise filtering, morphological operations such as closing and opening of an image, thresholding and image subtraction. The colors of the convective extracted image are compared against the color bar provided on the image to estimate the reflectivity values.

DWR image contains seventeen different reflectivity values, and each value represents the different amount of Reflectivity information. The Reflectivity value in DWR ranges from $20 \mathrm{dBZ}$ to $65 \mathrm{dBZ}$ in steps of $2.5 \mathrm{dBZ}$. The estimated reflectivity value from the DWR image using ANN has the continuous values 20 $\mathrm{dBZ}$ to $65 \mathrm{dBZ}$. The value is rounded off to the nearest value provided on the color bar chart. The Reflectivity value for each color combination is tabulated as shown in Table 1. 


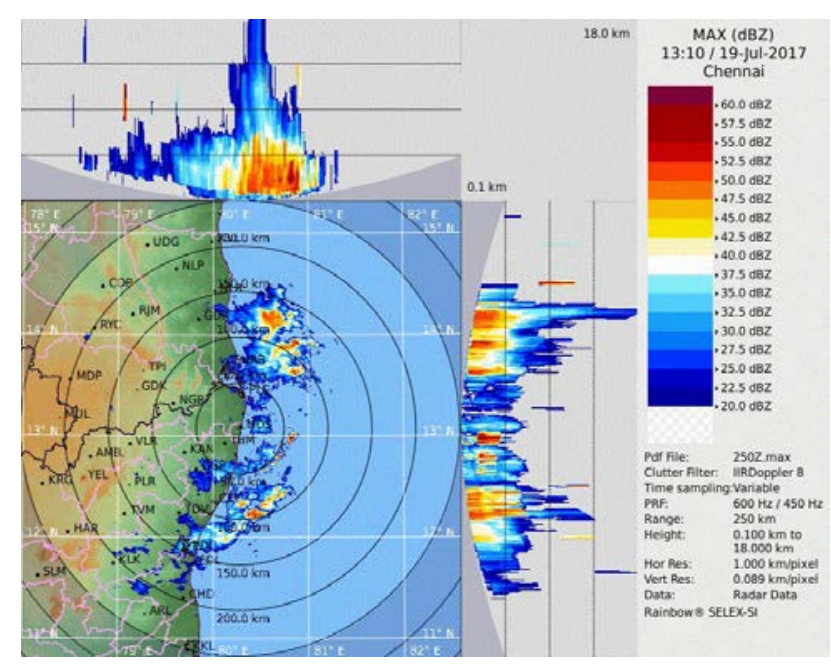

Figure 2. DWR Reflectivity image from IMD Chennai

$\left.\begin{array}{cc}\begin{array}{c}\text { Table 1. Color composite of Reflectivity values } \\ \hline \text { Reflectivity (in dBZ) }\end{array} & \text { Color values (RGB) } \\ \hline 20.0 & {\left[\begin{array}{lll}0 & 0 & 0.6\end{array}\right]} \\ 22.5 & {\left[\begin{array}{lll}0 & 0 & 0.8\end{array}\right]} \\ 25.0 & {\left[\begin{array}{lll}0 & 0.2 & 1\end{array}\right]} \\ 27.5 & {\left[\begin{array}{lll}0 & 0.4 & 1\end{array}\right]} \\ 30.0 & {\left[\begin{array}{lll}0 & 0.6 & 1\end{array}\right]} \\ 32.5 & {\left[\begin{array}{lll}0.2 & 0.8 & 1\end{array}\right]} \\ 35.0 & {\left[\begin{array}{llll}0.4 & 1 & 1\end{array}\right]} \\ 37.5 & {\left[\begin{array}{lll}1 & 1 & 1\end{array}\right]} \\ 40.0 & {\left[\begin{array}{lll}1 & 1 & 0.8\end{array}\right]} \\ 42.5 & {\left[\begin{array}{lll}1 & 1 & 0\end{array}\right]} \\ 45.0 & {\left[\begin{array}{lll}1 & 0.8 & 0\end{array}\right]} \\ 47.5 & {\left[\begin{array}{lll}1 & 0.4 & 0\end{array}\right]} \\ 50.0 & {\left[\begin{array}{lll}1 & 0.2 & 0\end{array}\right]} \\ 52.5 & {\left[\begin{array}{lll}0.8 & 0 & 0\end{array}\right]} \\ 55.0 & {\left[\begin{array}{lll}0.6 & 0 & 0\end{array}\right]} \\ 60.0 & {\left[\begin{array}{lll}0.6 & 0 & 0.2\end{array}\right]}\end{array}\right]$

\section{RESULTS AND DISCUSSIONS}

The reflectivity value of an image is represented by a unique combination of color values in the RGB model. Test patterns were generated with $10 \%$ deviation in the color composite. The test patterns are used to train, validate and test the network [21]. The networks have three inputs for which Red, Green, and Blue color values at a certain location are given as inputs. It has one output which is used to classify the 17 different reflectivity values [22]. In order to save time, memory and complexity of the network, the number of hidden layers used in this model is three. The block diagram of the neural network model is shown in Figure 3.

The neural network is trained with different types of back propagation algorithms such as variable learning rate backpropagation, Levenberg-Marquardt, One step secant, scaled conjugate gradient, and resilient back propagation [23]. The network is provided with 5100 different samples for training and testing. Out of which $80 \%$ of the samples is used for training the network, $10 \%$ of the samples are used for testing the network and the remaining $10 \%$ of the samples are used for validating the network. The results are compared over repeated iterations by shuffling the training sample values. The error histogram is a plot between error value and the number of instances the error has occurred. The error histogram of 20 bins is plotted as shown in Figure 4. The center of the histogram has the minimum error and the error increases as we move away from the center. The second plot which is trained with Levenberg-Marquardt has minimum error compared to the other algorithms. The plot shows that $99 \%$ of the samples fall within the range of \pm 1 which falls within the tolerable error limit of \pm 1.25 . The reflectivity values will be in steps of $2.5 \mathrm{dBZ}$. Any value falls within the range of $2.5 \mathrm{dBZ}$ will not be considered as an error. For example, the reflectivity range of $25 \mathrm{dBZ}$ is from $23.75 \mathrm{dBZ}$ to $26.25 \mathrm{dBZ}$. 


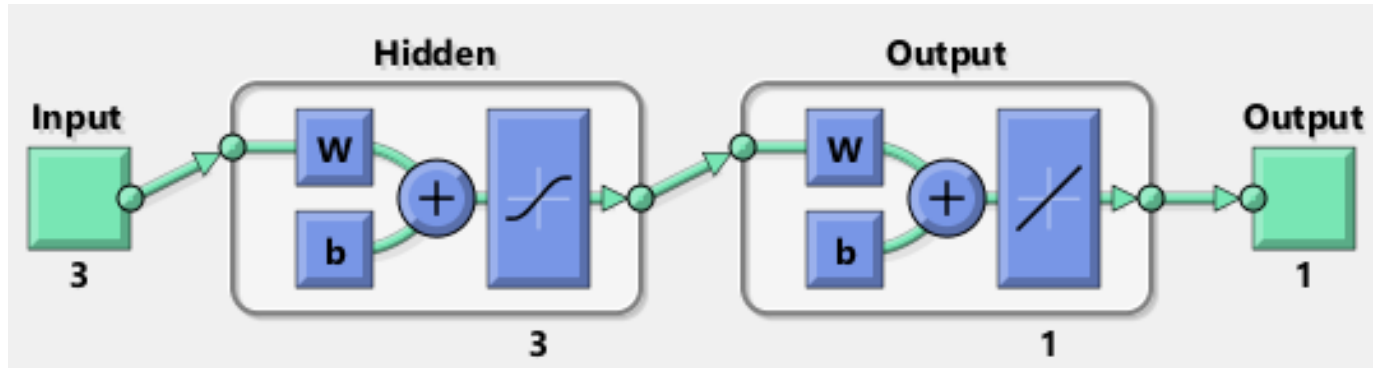

Figure 3. Block diagram of Neuron network model

The dashed line the regression plots indicates the outputs. The best possible fit between network outputs and desired targets is indicated by a solid dash line. The relation between outputs and targets is indicated by the regression value. The regression plot gives information about how close the output of your model is to the actual target values. The network outputs have a strong linear relation to desired targets if the value of Regression coefficient approaches unity. If the value of regression coefficient approaches zero, the relation between output and targets cannot be predicted. The regression plots for GDX, LM, OSS and SCG model is shown in Figure 5. The regression values for the GDX, LM, OSS, and SCG are 0.98697, 0.99812, 0.99346 and 0.98499 respectively. The LM backpropagation algorithm shows better performance compared with the other three models.

The performance plot is a plot between Mean Square Error (MSE) and the number of epochs. MSE is the average squared difference between outputs and targets. MSE of Zero implies no error. As the training process progresses, the MSE value reduces. When the MSE value is reduced to a minimum value, the training stops and the network are validated with the samples. In the validation phase, if the network behaves properly, then the training stops and it is ready for testing. The MSE values for GDX, LM, OSS, and SCG are 3.8841, $0.4964,1.8942$ and 4.4903 respectively. The LM shows better performance compared to other methods based on MSE.

In the previous work, Refelctivity extraction over a location is performed using different distance measures such as Euclidean, Standard Euclidean, City block, Minkowski, Chebychev, Mahalanobis, cosine and correlation [24, 25]. Color classification accuracy, using conventional distance measures is $95 \%$ for a standard Euclidean distance over different color spaces such as RGB, HSV, YCbCr and La*b*. The accuracy percentage for different standard distance measures and different neural network algorithms are tabulated in Table 2. The accuracy percentage values tabulated in Table 2 are the average values of repeated iteration. Reflectivity extraction using neural networks provides better results compared with the previous work.

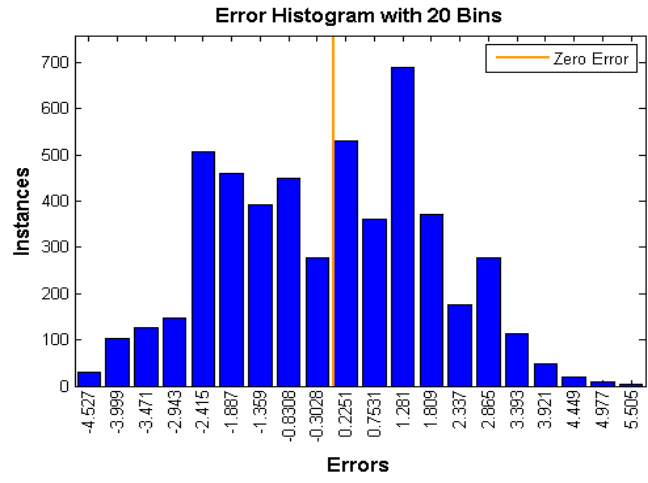

(a)

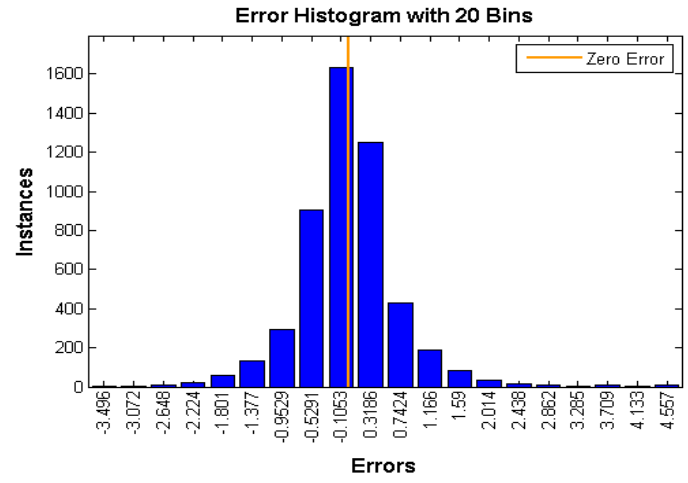

(b)

Figure 4. Error histogram plots (a) GDX (b) LM (c) OSS (d) SCG 


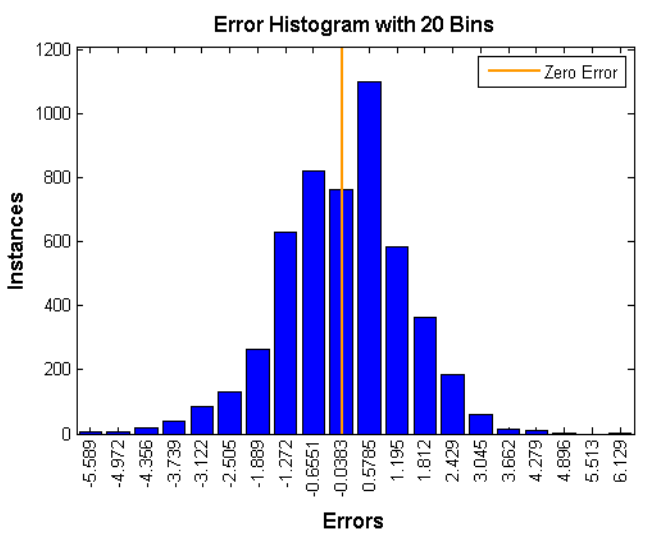

(c)

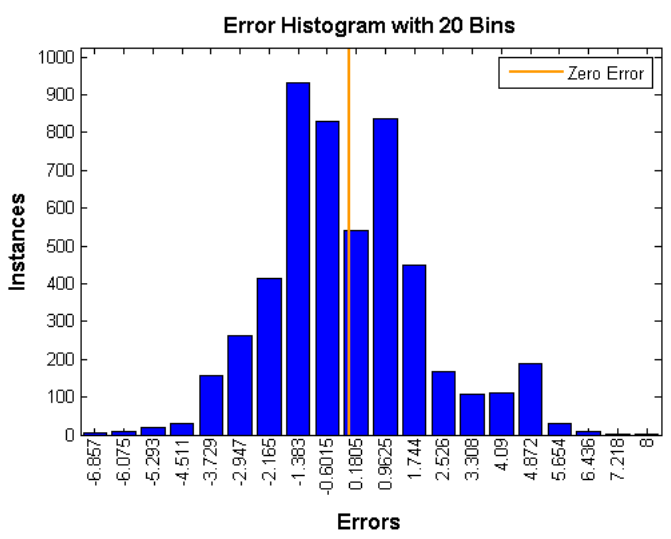

(d)

Figure 4. Error histogram plots (a) GDX (b) LM (c) OSS (d) SCG

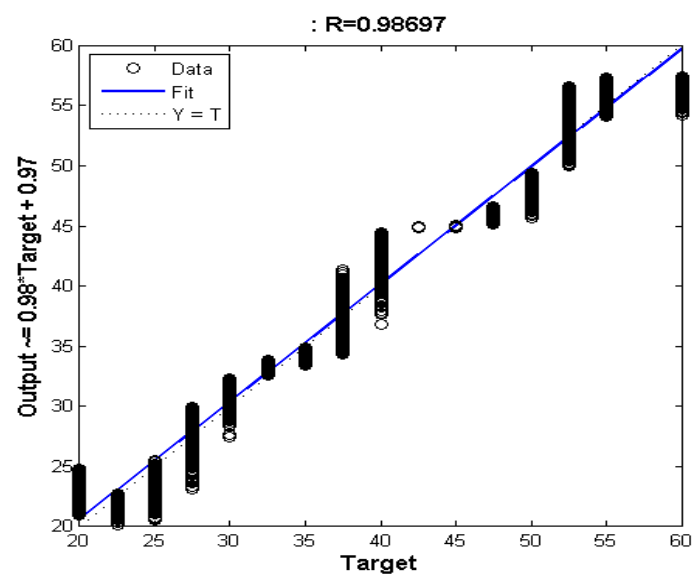

(a)

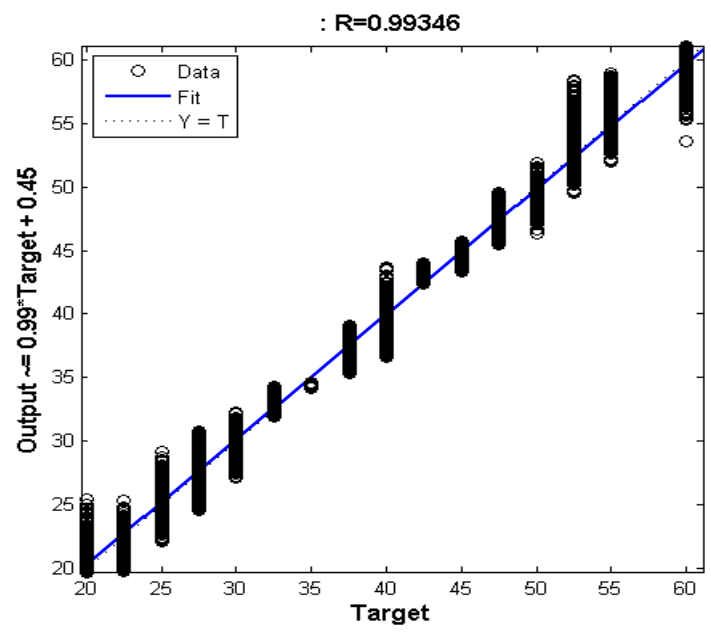

(c)

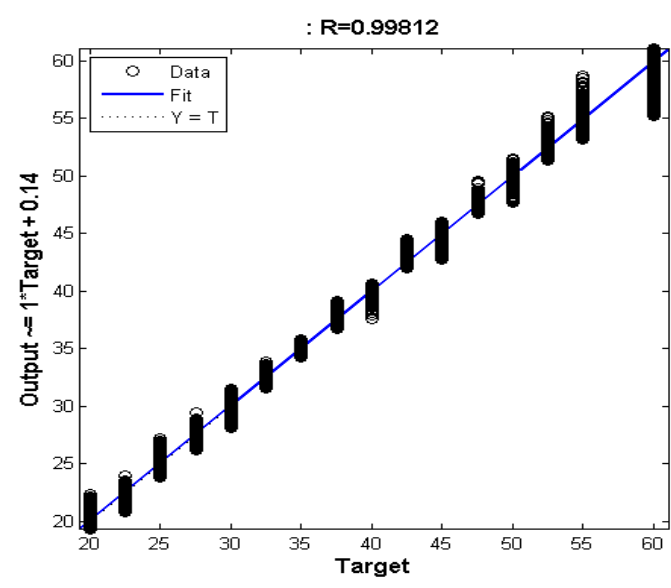

(b)

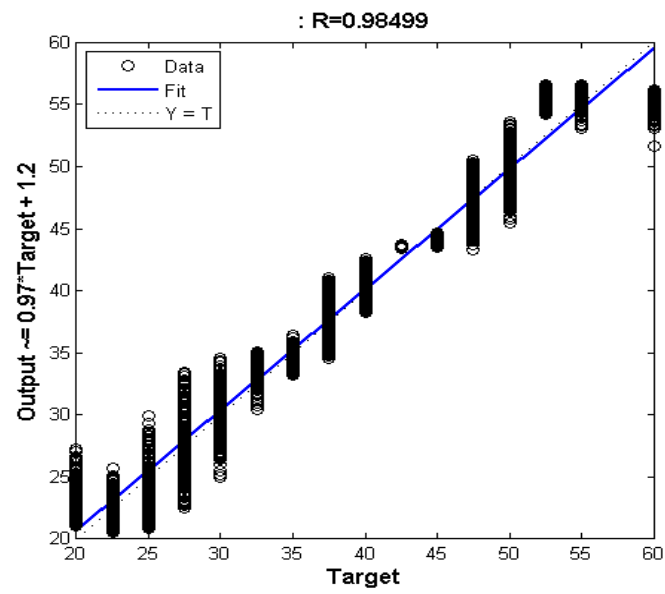

(d)

Figure 5. Regression plots (a) GDX (b) LM (c) OSS (d) SCG 


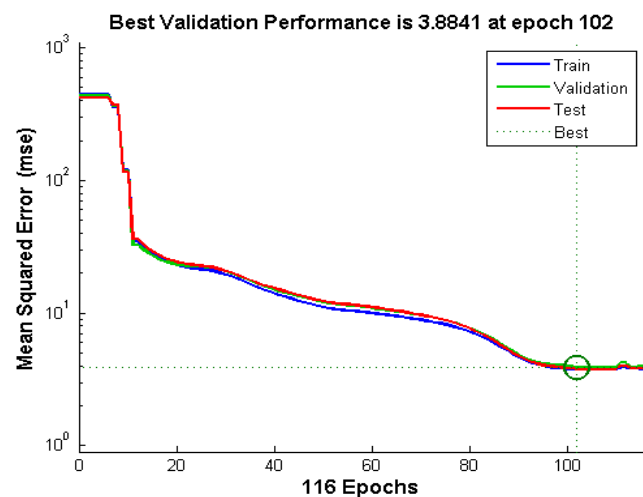

(a)

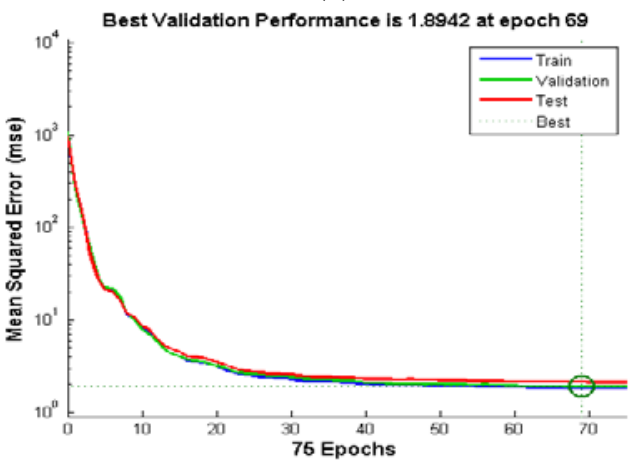

(c)

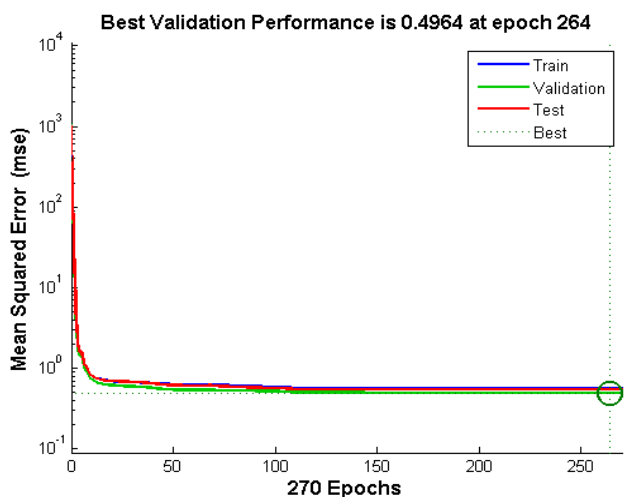

(b)

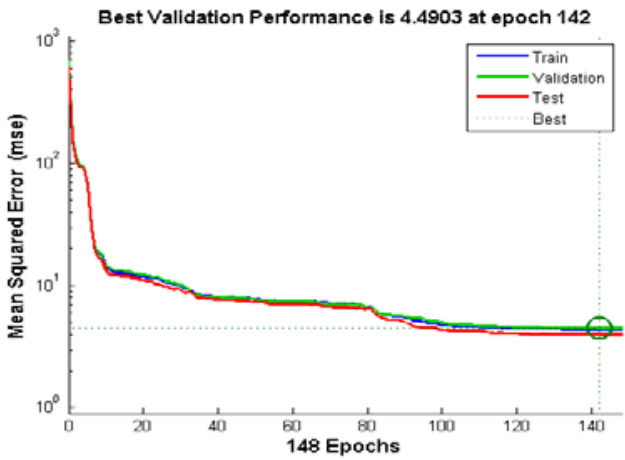

(d)

Figure 6. Performance Curves (a) GDX (b) LM (c) OSS (d) SCG

Table 2. Accuracy Percentage in RGB Space for Different Distance Metrics

\begin{tabular}{ccc}
\hline Method & & \% of Accuracy \\
\hline & Euclidean & 6.20 \\
& Seuclidean & 6.09 \\
& Cityblock & 6.29 \\
Distance Measures & Minkowski & 6.19 \\
& Chebychev & 6.21 \\
& Mahalanobis & 13.22 \\
& Cosine & 25.73 \\
& Correlation & 41.53 \\
& GDX & 13.18 \\
Neural Network Methods & LM & $\mathbf{1 . 8 2}$ \\
& OSS & 4.82 \\
& SCG & 7.22 \\
\hline
\end{tabular}

\section{CONCLUSION}

In this paper, extraction of the Reflectivity parameter from the DWR image is done with the help of artificial neural networks. The network is provided with 5100 sample inputs to classify the 17 different target outputs. The network is trained with different types of backpropagation algorithms such as LM, GDX, OSS, and SCG. The network trained with LM gives better performance and regression values. Even though the regression value changes for every iteration, LM proves to be the best compared to the other methods. LM presents better accuracy compared to the traditional standard distant measures.

\section{ACKNOWLEDGEMENT}

We are thankful to the "Atmospheric Remote Sensing and Advanced Signal Processing", Centre of Excellence at Department of ECE, S V University, Tirupati for providing necessary resources and financial support to carry out the present work. We are thankful to Dr. M.S.Arunachalam, PDF in IIT Chennai for helping me in my research work. 


\section{REFERENCES}

[1]. W. Zhuo, Z. G. Cao, and Y. Xiao, "Cloud classification of ground-based images using texture-structure features", Journal of Atmospheric and Oceanic Technology, vol. 31, no. 1, pp. 79-92, 2014.

[2]. Al Bovik, Ed., Handbook of Image and Video Processing. Academic, New York, 2000.

[3]. Niblack W., Berber R., Equitz W., Flickner M., Glasman E., Petkovic D., and Yanker P., "The QBIC project: Quering images by content using color, texture and shape”. in Proc. SPIE Storage and Retrieval for Image and Video Databases, 1994, pp. 172-187.

[4]. Rajithkumar B K , H.S. Mohana, "Template Matching Method for Recognition of Stone Inscripted Kannada Characters of Different Time Frames Based on Correlation Analysis”, International Journal of Electrical and Computer Engineering (IJECE), Vol. 4, No. 5, October 2014, pp. $719 \sim 729$

[5]. Nurdan Akhan Baykan, Nihat Y1lmaz, "Mineral identification using color spaces and artificial neural networks", Computers \& Geosciences Elsevier, 2010, pages 91-97.

[6]. Nader Jamali Soufi Amlashi , Amin Shahsavari, Alireza Vahidifar, Mehrzad Nasirian, ” Nonlinear System Identification of Laboratory Heat Exchanger Using Artificial Neural Network Model”, International Journal of Electrical and Computer Engineering (IJECE), Vol.3, No.1, February 2013, pp. 118 128

[7]. Dr. Meenakshi Sharma \& Anjali Batra, “Analysis of Distance Measures in Content Based Image Retrieval”, Global Journal of Computer Science and Technology, Volume 14, Online ISSN: 0975-4172, Year 2014.

[8]. Ioka M., "A method of defining the similarity of images on the basis of color information”, Technical Report RT0030, IBM Research, Tokyo Research Laboratory, Nov. 1989

[9]. Aisyah Hartini Jahidin, Mohd Nasir Taib, Nooritawati Md Tahir, Megat Syahirul Amin Megat Ali, "IQ Classification via Brainwave Features: Review on Artificial Intelligence Techniques", International Journal of Electrical and Computer Engineering (IJECE), Vol. 5, No. 1, February 2015, pp. 84 91 ISSN: 2088-8708

[10]. Daniel Graupe, "Principles of Artificial Neural Networks", World Scientific Publishing Co. Pte ltd., Vol 6, 2007

[11]. K.K. Bhoyar, O.G. Kakde, "Skin Color Detection Model Using Neural Networks and its Performance Evaluation”, Journal of Computer Science, ISSN 1549-3636, Pages 963-968, 2010.

[12]. Saurabh Karsoliya, "Approximating Number of Hidden layer neurons in Multiple Hidden Layer BPNN Architecture”, International Journal of Engineering Trends and Technology- Volume 3, Issue6- 2012.

[13]. Taravat, F. F. Del, C. Cornaro, and S. Vergari, "Neural networks and support vector machine algorithms for automatic cloud classification of whole-sky ground-based images,” IEEE Geoscience and Remote Sensing Letters, vol. 12, no. 3, pp. 666-670, 2014.

[14]. Hanaa M. Mushgil, Dr. Haithem A. Alani, Dr. Loay E. George, “ Comparison between Resilient and Standard Back Propagation Algorithms Efficiency in Pattern Recognition”, International Journal of Scientific \& Engineering Research, ISSN 2229-5518, Volume 6, Issue 3, March-2015.

[15]. M R Narasinga Rao, Deepthi Gurram, Sai Mahathi Vadde, Sathish Tallam, N. Sai Chand, L. Kiran, “A Predictive Model for Mining Opinions of an Educational Database Using Neural Networks”, International Journal of Electrical and Computer Engineering (IJECE), Vol. 5, No. 5, October 2015, pp. 1158 1163 ISSN: 2088-8708

[16]. Li Xinhua,Yu Qian, “ Face Recognition based on Deep Neural Network”, International Journal of Signal Processing, Image Processing and Pattern Recognition, Vol.8, No.10, pp.29-38, 2015.

[17]. Vu N.P. Dao, Rao Vemuri, “A Performance Comparison of Different Back Propagation Neural Networks Methods in Computer Network Intrusion Detection”, Differential Equations and Dynamical Systems, 2002.

[18]. http://www.imd.gov.in/pages/radar_main.php?adta=chn.

[19]. P. Anil Kumar(Research Scholar), Prof.B. Anuradha and M. Arunachalam Srinivasan, "Convective cloud parameter retrieval from Doppler Weather Radar MAX(Z) product using Image Processing Technique”, National Conference on ENVIRONMENTAL POLLUTION AND ITS IMPACT ON REGIONAL CLIMATE CHANGE (EPRCC-2016), 11th \& 12th March, 2016, Department of Physics, Sri Krishnadevaraya University, Anantapur.

[20]. Puli Anil Kumar, Dr. B. Anuradha, "Determination of convective cloud parameters using WRF and DWR data", International Journal of Modern Electronics and Communication Engineering (IJMECE), ISSN: 2321-2152, Volume No.-4, Issue No.-3, May, 2016.

[21]. Santaji Ghorpade, Jayshree Ghorpade and Shamla Mantri, "Pattern Recognition Using Neural Networks", International Journal of Computer Science \& Information Technology (IJCSIT), Vol 2, No 6, December 2010.

[22]. Selvarajah, S., Kodituwakku. S.R., "Analysis and Comparison of Texture Features for Content Based Image Retrieval”, International Journal of Latest Trends in Computing (E-ISSN: 2045-5364), vol. 2 (1), 2011, 108-113.

[23]. QI Yingjian, LUO Siwei, LI Jianyu, HUANG Huakun, "USING NEURAL NETWORK IN COLOR CLASSIFICATION", Proceedings of IEEE TENCON, 2002.

[24]. P. ANIL KUMAR, Dr. B. ANURADHA, Dr. M.S. Arunachalam, “ Extraction of Time Series Convective Cloud Profile from Doppler Weather Radar MAX (Z) Product Using A Novel Image Processing Technique”, International Journal of Advance Engineering and Research Development, Volume 4, Issue 7, July -2017.

[25]. Puli Anil Kumar, Dr. B. Anuradha, “ Estimating Reflectivity of DWR Images by Analysing Different Colour Spaces through Distance Measures”, Advances in Computational Sciences and Technology, ISSN 0973-6107 Volume 10, Number 8, 2017, pp. 2191-2200 